\title{
Neurocytoma of the pituitary gland: A case report and literature review
}

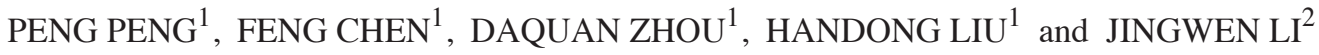 \\ Departments of ${ }^{1}$ Neurosurgery and ${ }^{2}$ Oncology, Xiangyang Central Hospital, \\ Hubei University of Arts and Science, Xiangyang, Hubei 441021, P.R. China
}

Received December 9, 2014; Accepted January 22, 2015

DOI: $10.3892 /$ br.2015.430

\begin{abstract}
Extraventricular neurocytomas (EVN) are rare central nervous system tumors, often located within the cerebral hemisphere. The present study reports a case of a 56-year-old male patient with bitemporal hemianopsia. Computed tomography and magnetic resonance imaging revealed a tumor in the sellar region. The tumor was totally excised. Postoperative histological examination of the tumor demonstrated that synaptophysin, chromogranin-A and neuron-specific enolase were positive, while luteinizing hormone, follicle-stimulating hormone, growth hormone, prolactin, adrenocorticotropic hormone, thyroid-stimulating hormone, glial fibrillary acidic protein, S-100, nestin and epithelial membrane antigen were negative, which were the main pathological features of neurocytomas. This is the fourth case of EVN located in the sellar region reported. The associated studies are also reviewed.
\end{abstract}

\section{Introduction}

Central neurocytomas (CNC), World Health Organization (WHO) grade II, are relatively rare central nervous system (CNS) tumors that are often located in the ventricular system, particularly the septum, the third ventricle and the lateral ventricles. However, this entity has also been reported outside the ventricular system, and thus, was termed as extraventricular neurocytoma $(\mathrm{EVN})$, which exhibits a wide variability in respect of morphological characteristics, cellularity and proliferation rate and are more frequently associated with aggressive histological features (1). The present study reports an unusual case of sellar neurocytoma and reviews the associated studies.

Correspondence to: Mrs. Jingwen Li, Department of Oncology, Xiangyang Central Hospital, Hubei University of Arts and Science, 39 Jingzhou Street, Xiangyang, Hubei 441021, P.R. China

E-mail: drzhyi@gmail.com

Key words: extraventricular, neurocytoma, pituitary, sellar

\section{Case report}

A 56-year-old male patient with a past history of hypertension and left ocular trauma presented with a headache following a head injury. A neoplasm of the sellar region was occasionally identified from the computed tomography (CT) that was performed in a local hospital. Physical examination on admission revealed a well-developed and nourished male. There were no signs of hypophysial dysfunction on admission. Ophthalmological tests disclosed bitemporal hemianopsia and decreased visual acuity in the left eye (0.2). Asymmetry in bilateral pupillary size was found; 3.5 and $2.5 \mathrm{~mm}$ in the left and right, respectively. Direct pupillary light reflex of the left eye was slower than the right, while the indirect pupillary light reflex of both was the same. The remainder of the neurological examination was normal.

Routine laboratory tests revealed a mildly decreased cortisol (8 a.m.) level (6.8 $\mu \mathrm{g} / \mathrm{dl}$; normal range, 8.7-22.4 $\mu \mathrm{g} / \mathrm{dl})$. The remaining pituitary hormone was normal.

A CT scan revealed a sellar region tumor without calcification. Coronal T1-weighted magnetic resonance imaging (MRI) (Fig. 1A) of the patient showed a well-defined dumbbell-shaped pituitary mass $(3 \times 2 \mathrm{~cm})$ that was hypointense to the brain parenchyma with compression of the optic chiasm and the third ventricle. On sagittal T2-weighted MRI (Fig. 1B) studies, the signal was isointense to the surrounding grey matter with infiltration of the sphenoid sinus and suprasellar space. Following the intravenous administration of gadolinium (Fig. 1C), the neoplasm was enhanced heterogeneously. Magnetic resonance angiography depicted that the tumor closely adhered to the bilateral internal carotid arteries, without encasement. Therefore, pre-surgical radiological diagnosis was possibly a pituitary macroadenoma.

Following successful endotracheal intubation using general anesthesia, the patient was in the supine position with head rightward by $\sim 45$ degrees. An extended left side pterional cranioctomy was performed, the carotid cistern and optic chiasm cistern were opened, the left internal carotid artery and optic nerve were separated and finally the tumor was exposed under the surgical microscope. The tumor was of a soft texture, was red in color and there was no abundant bleeding. Satisfactory clearance of the neoplasm was achieved by piecemeal resection without postoperative complications. 

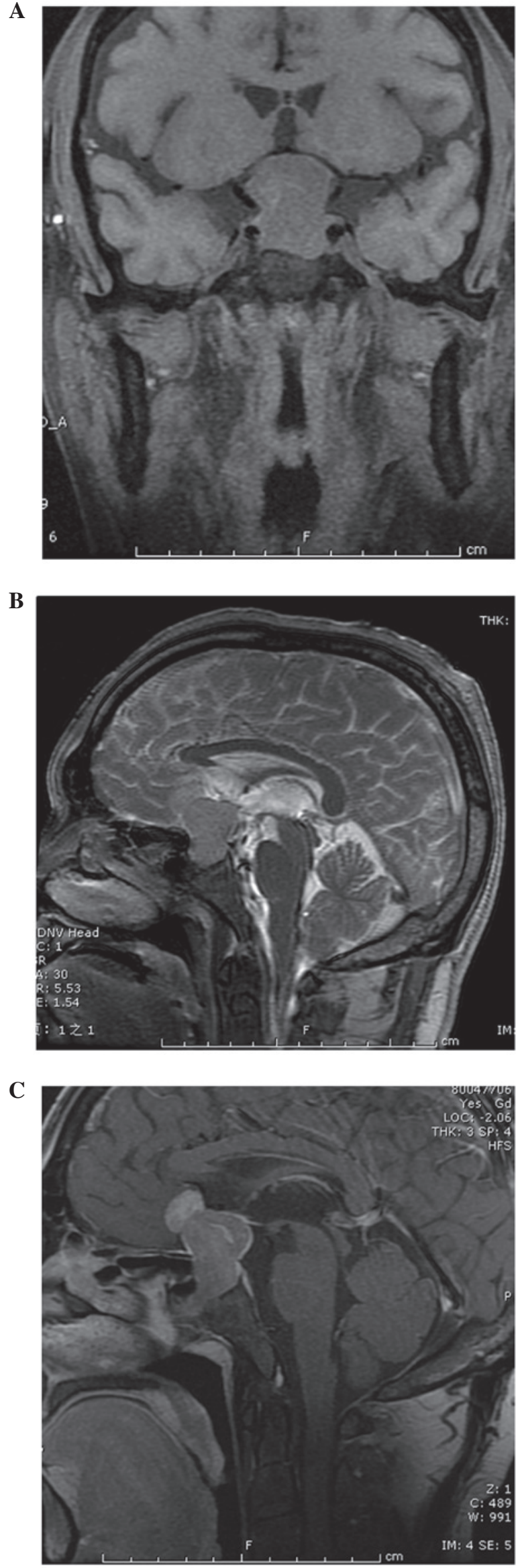

Figure 1. (A) Coronal T1-weighted and (B) sagittal T2-weighted magnetic resonance images showing a well-defined dumbbell shape pituitary mass with compression of the optic chiasm and the third ventricle. (C) he neoplasm is enhanced heterogeneously.
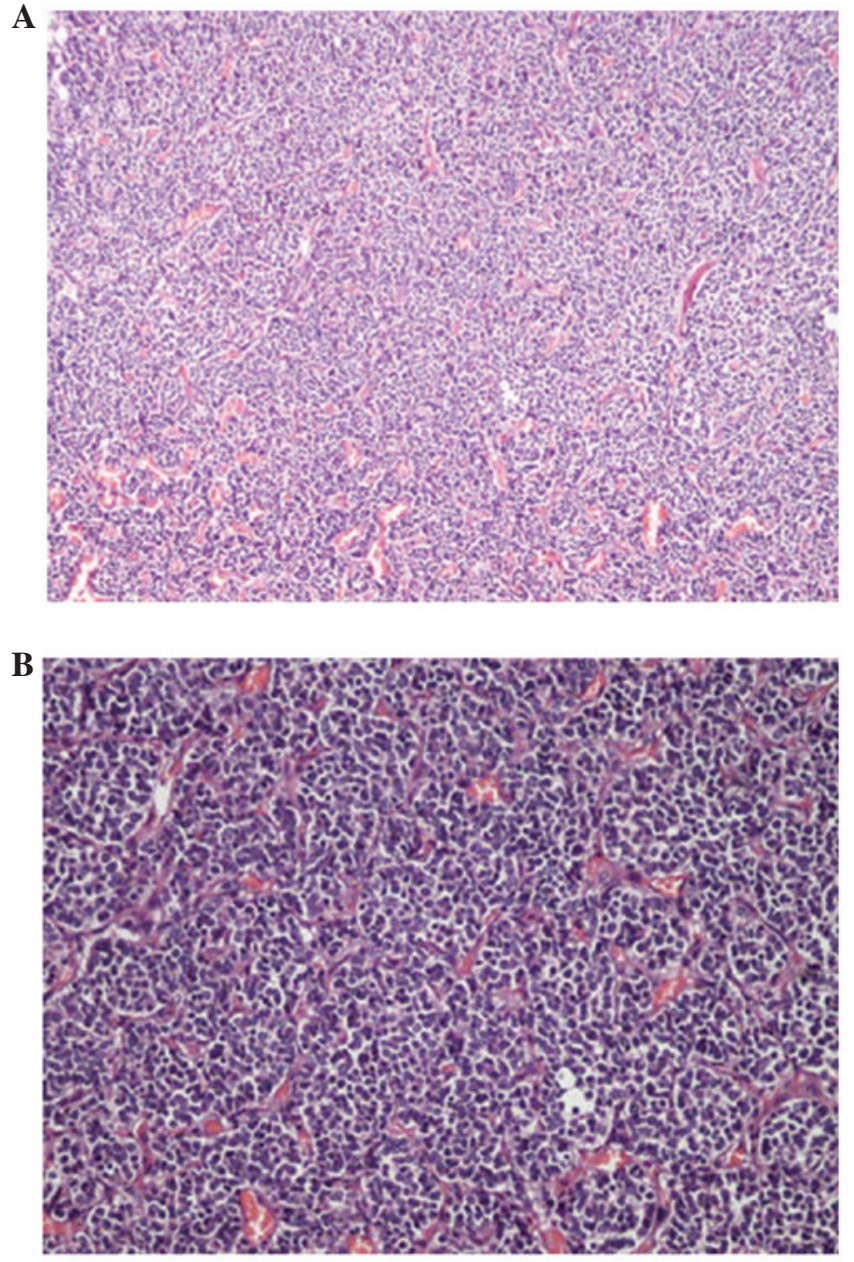

Figure 2. Histology of the tumor [hematoxylin and eosin staining; magnification, (A) x100; and (B), x200]. A region of the tumor consisted of a monotonous population of tumor cells with rounded nuclei, finely stippled chromatin and inconspicuous nucleoli, along with clearing of the cytoplasm. Thin-walled capillary-sized vessels are arranged in a linear arborizing pattern.

Histological examination of the tumor revealed a neurocytoma composed of uniform sheets, small-to-medium-sized tumor cells with round regular nuclei and salt-and-pepper chromatin, along with scant cytoplasm (Fig. 2A and B). These cells are closely arrayed, with a background of filamentous matrix having a neuropil-like quality. Thin-walled capillary-sized vessels, arranged in a linear arborizing pattern, were observed. Immunohistochemically, synaptophysin (SYN), chromogranin-A (CGA) and neuron-specific enolase (NSE) were positive, while luteinizing hormone ( $\mathrm{LH})$, follicle-stimulating hormone $(\mathrm{FSH})$, growth hormone $(\mathrm{GH})$, prolactin (PRL), adrenocorticotropic hormone (ACTH), thyroid stimulating hormone (TSH), glial fibrillary acidic protein (GFAP), S-100, nestin and epithelial membrane antigen (EMA) were negative and Ki-67 proliferation index was at a level of 0-1\%. Finally, the WHO grading of the entity was II.

\section{Discussion}

CNC, first named by Hassoun in 1982, accounts for $0.25-0.5 \%$ of all CNS tumors and usually affects young adults of approximately thirty years of age, with a range between eight days and 67 years $(2,3)$. This type of entity is usually located within 
the ventricular system, particularly the foramina of Monro. However, central neurocytoma-like neoplasms that arise within brain parenchyma have been described in different studies. EVN, first reported in 1989, is generally observed to affect children and young adults, with cases ranging from 5 to 76 years (median, 34 years), without gender predominance (4). According to previous studies, the cerebral hemisphere, thalamus, cerebellum, pons, amygdala, pineal gland, retina and spinal cord have been documented as the location of EVN. The present study is the fourth case of EVN in the sellar region $(5,6)$.

Analyzing the four case reports for the clinical manifestation, decreased visual acuity and hemianopsia are common. Endocrine tests are usually normal.

Although the radiographic differentiation of EVNs and other tumors has not been well described, EVNs have their own imaging characteristic. Generally, EVNs are discrete, sometimes large, complex and variably enhancing masses (5). The content of EVNs can be solid, cystic or both, with or without calcification. On T1-weighted MRI images, the signal of solid mass was isointense or slightly hypointense, while on T2 hyperintense images, there is a moderate to strong enhancement following gadolinium administration. However, Aralasmak et al (7) reported one case without enhancing. Therefore, they believed that enhancement is not always necessary for EVNs. Peritumoral edema can be observed in certain cases and hemorrhage of tumors was sporadically reported (8). Proton MR spectroscopic studies of EVNs demonstrate the following typical changes: Elevated choline, decreased $\mathrm{N}$-acetylaspartate and decreased creatine $(5,9)$. When a parenchymal tumor with cystic necrosis, calcification and extensive enhancement in a young patient was encountered, EVN should be in the range of differential diagnosis.

The diagnosis of EVNs was based on the immunohistochemical features, given the overlap of clinical, radiological and histological features with those of other CNS tumors, particularly oligodendroglioma. SYN- and NSE-positive staining suggests a diagnosis of neurocytoma; even NSE is not specific for neurocytoma. A GFAP immunopositive reaction is usually observed in gliomas, such as oligodendroglioma, while negative is in the majority of EVNs. Zhu et al (10) found that neuronal nuclear antigen (NeuN) was positive in almost all cases of CNC. Therefore, they believed that NeuN can be applied as another reliable marker of neurocytoma. From a genetic perspective, without the $1 p / 19 q$ deletion and the absence of p53 immunoexpression, O6-methylguanine-DNA methyltransferase promoter methylation and low frequency of epidermal growth factor receptor gene amplification are the characteristics of EVNs, which differentiate it from oligodendrogliomas (11). Electron microscopic evaluation is necessary to confirm the diagnosis when encountering an ambiguous immunophenotype. In the patient of the present study, NSE and SYN stained positive, while GFAP, S-100, nestin and EMA were negative, suggesting the diagnosis of a neurocytoma. Expression of CGA suggests this entity was admixed with ganglion cells. Absence of LH, FSH, GH, PRL, ACTH and TSH excludes the diagnosis of a pituitary adenoma.
Radiotherapy and chemotherapy have played an important role in the management of patients with EVNs. Rades et al (12) have documented that postoperative radiotherapy improved the local control and survival rates of patients with incomplete resection. The optimal dose of radiotherapy appears to be 54-60 Gy (3). Gamma-knife surgery delivers high-dose radiation with minimal long-term side effects. Although the experience in chemotherapy is not rich, certain investigators have found it beneficial in recurrent EVNs that cannot be totally resected and failed in radiotherapy. von Koch et al (13) treated a 20-year-old female who underwent four subtotal resections, but exhibited an enlarged tumor, with procarbazine, lomustine and vincristine for chemotherapy. The tumor size started decreasing subsequent to two cycles of treatment and continued to shrink until it stabilized after five cycles. In the present case, gross total resection was performed and the patient did not receive radiotherapy or chemotherapy. A long-term follow-up workshop should be performed.

In conclusion, EVNs are relatively rare tumors. Differential diagnosis with other tumors, particularly oligodendrogliomas, is difficult as they share common clinical, radiological and histological features. Total excision should be the goal of treatment and adjuvant radiotherapy should be considered in the case of subtotal resection.

\section{References}

1. Brat DJ, Scheithauer BW, Eberhart CG and Burger PC: Extraventricular neurocytomas: Pathologic features and clinical outcome. Am J Surg Pathol 25: 1252-1260, 2001.

2. Sgouros S, Jackowski A and Carey MP: Central neurocytoma without intraventricular extension. Surg Neurol 42: 335-339, 1994.

3. Sharma MC, Deb P, Sharma S and Sarkar C: Neurocytoma: A comprehensive review. Neurosurg Rev 29: 270-285, 2006.

4. Ferreol E, Sawaya R and de Courten-Myers GM: Primary cerebral neuroblastoma (neurocytoma) in adults. J Neurooncol 7: 121-128, 1989.

5. Yang GF, Wu SY, Zhang LJ, Lu GM, Tian W and Shah K: Imaging findings of extraventricular neurocytoma: Report of 3 cases and review of the literature. AJNR Am J Neuroradiol 30: 581-585, 2009.

6. Wang Y, Tao R and Liu B: Response to: Extraventricular neurocytoma of the sellar region. Br J Neurosurg 27: 551-552, 2013.

7. Aralasmak A and Karaali K: Nonenhancing hypovascular extraventricular neurocytoma. AJNR Am J Neuroradiol 30: E117-E118, 2009.

8. Ritz R, Roser F, Bornemann A, Hahn U and Freudenstein D: Extraventricular neurocytoma presenting with intratumoral hemorrhage. Clin Neuropathol 24: 101-105, 2005.

9. Ueda F, Suzuki M, Matsui O and Uchiyama N: Automated MR spectroscopy of intra- and extraventricular neurocytomas. Magn Reson Med Sci 6: 75-81, 2007.

10. Zhu P, Yan F, Ma Y and Ao Q: Clinicopathological analysis of central and extraventricular neurocytoma: A report of 17 cases. J Huazhong Univ Sci Technolog Med Sci 30: 746-750, 2010.

11. Myung JK, Cho HJ, Park CK, Chung CK, Choi SH, Kim SK and Park SH: Clinicopathological and genetic characteristics of extraventricular neurocytomas. Neuropathology 33: 111-121, 2013.

12. Rades D, Fehlauer F, Lamszus K, Schild SE, Hagel C, Westphal M and Alberti W: Well-differentiated neurocytoma: What is the best available treatment? Neuro Oncol 7: 77-83, 2005.

13. von Koch CS, Schmidt MH, Uyehara-Lock JH, Berger MS and Chang SM: The role of PCV chemotherapy in the treatment of central neurocytoma: Illustration of a case and review of the literature. Surg Neurol 60: 560-565, 2003. 